\title{
Advanced controller design for uncertain linear systems with time-varying delays via augmented zero equality approach
}

\author{
YongGwon Lee ${ }^{1}$, Youngjae Kim ${ }^{1}$, Seungho Kim${ }^{1}$, Seunghoon Lee ${ }^{1}$, Myeongjin Park ${ }^{2}$, and \\ O. M. Kwon ${ }^{1}$
}

${ }^{1}$ Chungbuk National University

${ }^{2}$ Kyung Hee University - Global Campus

June 1, 2021

\begin{abstract}
This paper deals with the stability analysis and controller design for linear systems with time-varying delays and parameter uncertainties. By choosing appropriate augmented Lyapunov-Krasovskii functionals, a set of Linear Matrix inequalities is derived to get advanced feasible region of stability, and controller gain matrices which guarantee the asymptotic stability of the concerned systems within maximum bound of time-delays and its time-derivative. To further reduce the conservatism of stabilization criterion a recently developed mathematical technique which constructed a new augmented zero equality is applied. Finally, two numerical examples are utilized to show the validity and superiority of the proposed methods.
\end{abstract}

\section{Hosted file}

mmas_Lee-1.pdf available at https://authorea.com/users/417141/articles/524338-advancedcontroller-design-for-uncertain-linear-systems-with-time-varying-delays-via-augmentedzero-equality-approach 